\title{
Practical and Robust NMR-Based Metabolic Phenotyping of Gut Health in Early Life
}

\author{
Liene Bervoets, Johannes H. Ippel, Agnieszka Smolinska, Niels van Best, Paul H. M. Savelkoul,
} Monique A. H. Mommers, and John Penders*

Cite This: J. Proteome Res. 2021, 20, 5079-5087

Read Online

ABSTRACT: While substantial efforts have been made to optimize and standardize fecal metabolomics for studies in adults, the development of a standard protocol to analyze infant feces is, however, still lagging behind. Here, we present the development of a hands-on and robust protocol for proton ${ }^{1} \mathrm{H}$ NMR spectroscopy of infant feces. The influence of extraction solvent, dilution ratio, homogenization method, filtration, and duration of centrifugation on the biochemical composition of infant feces was carefully evaluated using visual inspection of ${ }^{1} \mathrm{H}$ NMR spectra in combination with multivariate statistical modeling. The optimal metabolomics protocol was subsequently applied on feces from seven infants collected at 8 weeks, 4, and 9 months of age. Interindividual variation was exceeding the variation induced by different fecal sample preparation methods, except for filtration. We recommend extracting fecal samples using water with a dilution ratio of

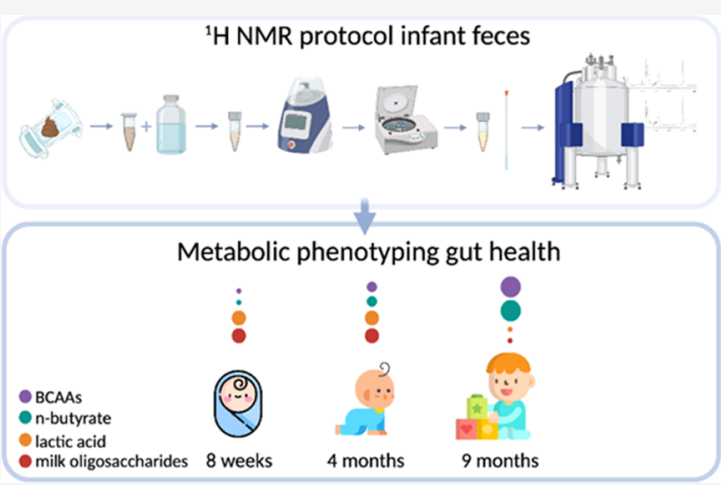
1:5 feces-to-water to homogenize using bead beating and to remove particulates using centrifugation. Samples collected from infants aged 8 weeks and 4 months showed elevated concentrations of milk oligosaccharide derivatives and lactic acid, whereas short-chain fatty acids (SCFAs) and branched-chain amino acids (BCAAs) were higher in the 9 month samples. The established protocol enables hands-on and robust analyses of the infant gut metabolome. The wide-ranging application of this protocol will facilitate interlaboratory comparison of infants' metabolic profiles and finally aid in a better understanding of infant gut health.

KEYWORDS: infant, feces, metabolomics, NMR spectroscopy, optimization, protocol

\section{INTRODUCTION}

Over the past decades, the role of the gut microbiome in health and disease has attracted increasing attention and is now a leading edge of scientific research. Microbial perturbations have been associated with a variety of noncommunicable diseases, including immune-mediated diseases such as allergies $^{1,2}$ and inflammatory bowel diseases. ${ }^{3,4}$ In this context, adequate development of the indigenous microbiome during infancy seems of particular importance for adequate maturation of the enteric mucosal tissues and immune system. ${ }^{5,6}$ Detailed insights into the development of not only the microbiota composition but also its function during infancy and early childhood are therefore pivotal to find new leads to manage and maintain health. ${ }^{7,8}$

Together with the rise of next-generation sequencing platforms for gut microbiome research, the development and application of multiomics approaches such as metagenomics, metatranscriptomics, metabolomics, and culturomics have also increased. ${ }^{9}$ Researchers can now analyze the taxonomic composition and structure of the gut microbiome but also study and verify the function of the microbiome and its association with health and disease. ${ }^{10}$
Feces is commonly used as a good proxy for examining gut health, especially in pediatric studies and large cohorts with a longitudinal research setting because its collection is easy and noninvasive. ${ }^{11}$ Feces consists of undigested food particles, microorganisms, metabolic byproducts of various processes, mucus, bile, bilirubin, water, minerals, salts, and gas. Fecal metabolites that circulate in the gut are a reflection of the complex interactions between nutrients, xenobiotics, microorganisms, and host immunity. ${ }^{11}$ Metabolomics, a tool to identify and quantify hundreds of low-molecular-weight metabolites, can be applied to phenotype an individual's health state and to discover biomarkers that may predict disease or response to treatment. ${ }^{12}$

While substantial efforts have been made to optimize and standardize fecal metabolomics for studies in adults, the development of a standard protocol to analyze infant feces is

Received: July 24, 2021

Published: September 30, 2021 
still lagging behind. ${ }^{13,14}$ Knowledge on the postnatal fecal metabolome is of particular interest as it can improve our understanding of early life exposures during a critical time window, infant gut health, and consequently the predisposition for chronic conditions later in life. ${ }^{12}$ However, the fecal metabolome of newborns is more complex to study than that of adults. First of all, the infant fecal metabolome changes rapidly over time due to gut microbiome development, immune maturation, and most importantly, the transition from milk feeding to solid foods. ${ }^{2,15}$ Second, infant stool consistency can vary substantially due to differences in food types. And finally, infant fecal samples usually have a small volume, making it difficult or even impossible to extract enough feces for metabolomics analyses. This illustrates the urgent need for a specialized and robust protocol to process infant fecal samples for untargeted metabolomics analysis.

Up to date, there is no study presented that describes the optimal treatment conditions of infant fecal samples for metabolic fingerprinting by proton $\left({ }^{1} \mathrm{H}\right)$ nuclear magnetic resonance (NMR) spectroscopy. The aim of this study was to develop a robust and feasible protocol for ${ }^{1} \mathrm{H}$ NMR-based metabolomics of infant feces. The development of this protocol was based on evaluating the influence of different sample preparation conditions on the fecal metabolic profile and selecting the most optimal conditions. In the end, our aim is to optimize this protocol such that the majority of metabolites will be effectively extracted, a wide range of compound classes (with the focus on short-chain fatty acids, SCFAs) will be covered, and that the procedure will not modify metabolites through chemical or physical means. To examine the feasibility and validity of the optimized protocol, it was subsequently applied to a study group of healthy newborns to monitor and quantify longitudinal changes in the fecal metabolome.

\section{METHODS}

\section{Subject Characteristics}

Infants previously enrolled in the LucKi Gut study, a longitudinal cohort study carried out in Maastricht, the Netherlands, ${ }^{16}$ were asked to participate in the LucKi metabolomics substudy after receiving pertinent information. Infants born preterm (less than 37 weeks of gestation) were excluded. The study was conducted in accordance with the ethical rules of the Helsinki Declaration and Good Clinical Practice and was approved by the Research Ethics Committee of Maastricht University Medical Center+ (METC 15-4$237.1 / \mathrm{ab}$ ). All parents gave written informed consent prior to inclusion in the study. Subject characteristics are outlined in Table S1 (Supporting Information). Five subjects were included to develop the optimal ${ }^{1} \mathrm{H}$ NMR method. Two additional subjects were included for monitoring and quantifying longitudinal changes in the fecal metabolome.

\section{Fecal Specimen Collection}

Fresh fecal samples were collected directly from the diaper into fecal collection tubes (Sarstedt AG \& Co. KG, Germany), which was accomplished by the parents at home. The collected material was immediately frozen at $-20{ }^{\circ} \mathrm{C}$ into a household freezer. Within 1 month, samples were delivered to the laboratory using a cool transport container (Sarstedt AG \& Co. $\mathrm{KG}$, Germany) to prevent thawing of samples during transport. Next, aliquots of $100 \mathrm{mg}$ feces were obtained per infant and stored at $-80{ }^{\circ} \mathrm{C}$ until further analysis was conducted.
Fecal Sample Preparation for ${ }^{1} \mathrm{H}$ NMR Method Development

Upon thawing, fecal samples collected from five infants aged between 4 and 9 months were subjected to different sample preparation conditions to determine the optimal sample preparation protocol (see Figure 1). A reference protocol

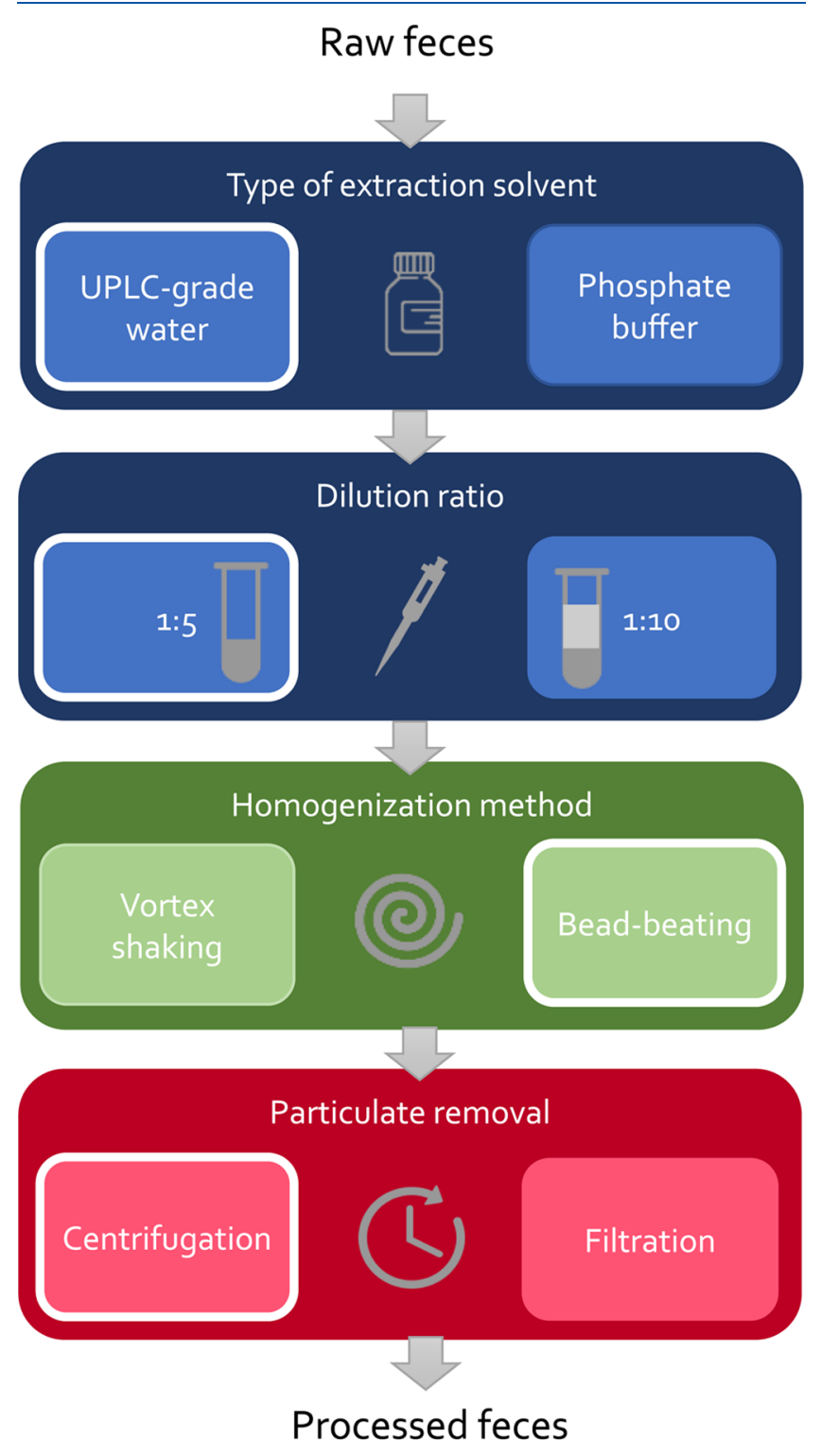

Figure 1. Schematic overview of different sample preparation steps for ${ }^{1} \mathrm{H}$ NMR-based metabolomics of infant feces. The impact of the type of extraction solvent, dilution ratio, homogenization method, duration of centrifugation, and filtration on the fecal ${ }^{1} \mathrm{H}$ NMR metabolite profile was examined. The different steps belonging to the optimized protocol are highlighted with white boxes.

was developed based upon established protocols for the handling of adult feces for NMR-based metabolomics. ${ }^{14,17}$ This protocol consisted of extracting the fecal sample with UPLC-grade water with a dilution ratio of 1:5 feces-to-water, vortex shaking for $20 \mathrm{~min}$ at $4{ }^{\circ} \mathrm{C}$ (Thermal Shake Lite, VWR, Radnor, PA), and centrifugation at $16000 \mathrm{~g}$ for $30 \mathrm{~min}$ at $4{ }^{\circ} \mathrm{C}$ (ROTINA 380 R Centrifuge, Germany). To assure the quality and assess the analytical variance of the data, a pooled sample was created by combining an equal volume of supernatants of 
A

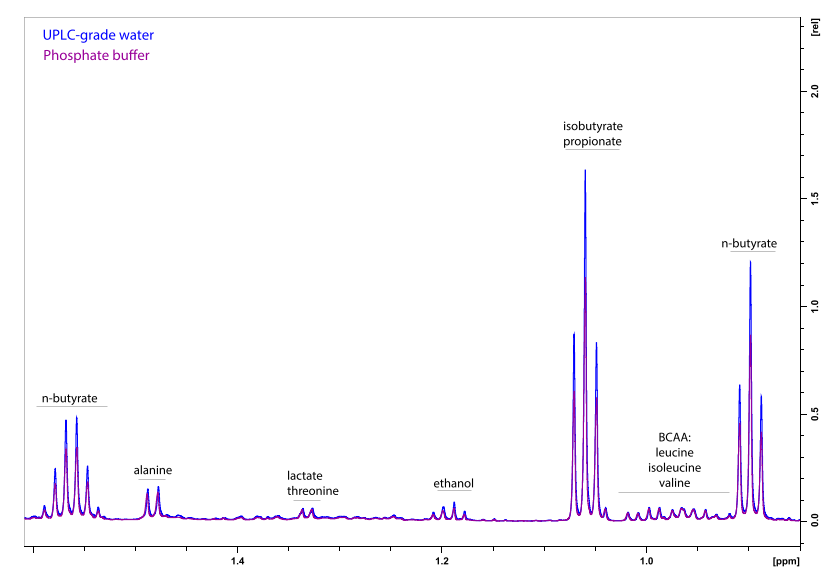

C

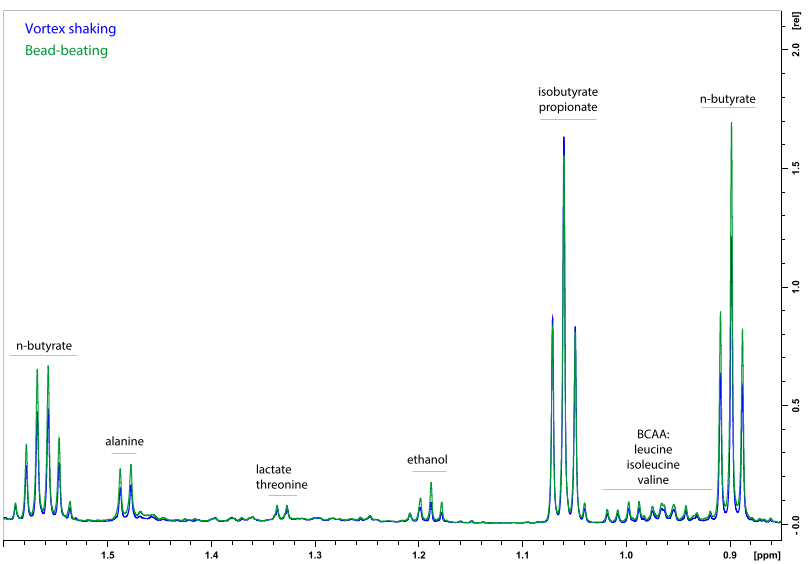

B

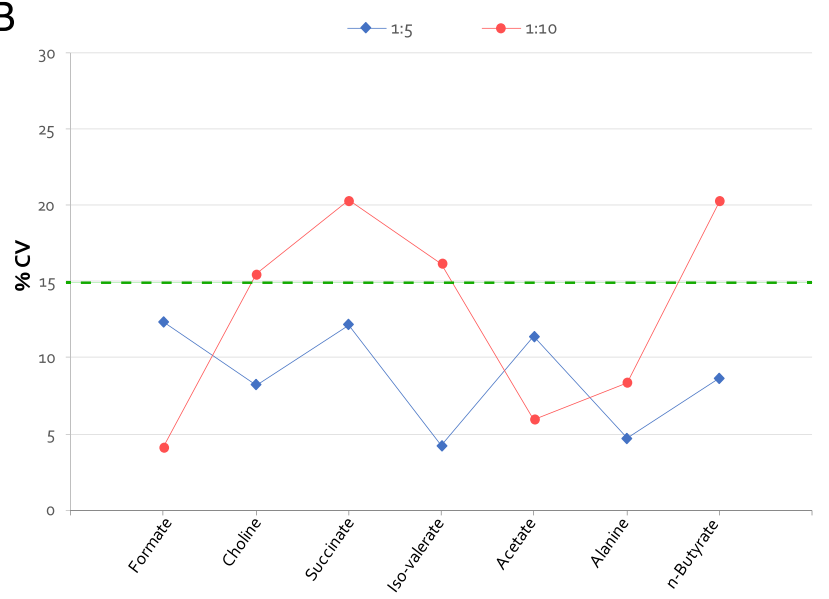

D

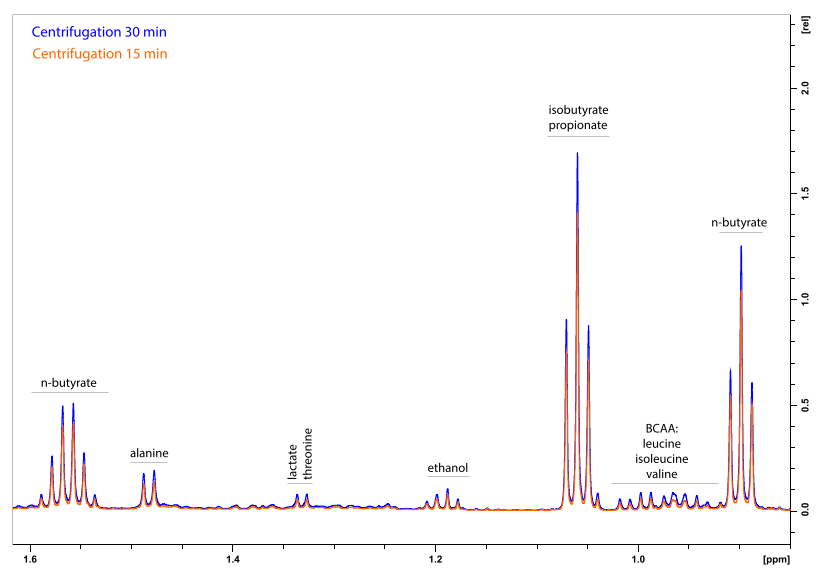

Figure 2. ${ }^{1} \mathrm{H}$ NMR spectra $(0.5-1.6 \mathrm{ppm})$ of infant fecal samples prepared with different extraction solvents (A), homogenization methods (C), and centrifugation duration times (D); and (B) the coefficient of variation (\%CV) of seven different fecal metabolites prepared with different water dilutions. The green dotted line illustrates the CV threshold of $15 \%$. $\%$ V was measured in duplicate.

the five individual samples. The impact of the type of extraction solvent was determined by diluting the samples with UPLC-grade water or phosphate buffer $(10 \mathrm{mM}, \mathrm{pH} 7.4$ at $25^{\circ} \mathrm{C}$ ) at two different dilution ratios, i.e., 1:5 and 1:10. The impact of homogenization was tested by comparing vortex shaking for $20 \mathrm{~min}$ at $4{ }^{\circ} \mathrm{C}$ to bead beating for $10 \mathrm{~s}$ at $4 \mathrm{~m} / \mathrm{s}$ (MP Fastprep-24 5 g, MP Biomedicals, Santa Ana, CA) using two zirconia beads of $0.5 \mathrm{~mm}$ (BioSpec Products Inc., Bartlesville, OK). The impact of different methods to remove particulate matter was examined by comparing centrifugation either for 15 or $30 \mathrm{~min}$ at $16000 \mathrm{~g}$ at $4{ }^{\circ} \mathrm{C}$ to filtration through a 3,10 , or $30 \mathrm{kDa}$ molecular weight centrifugal filter (Amicon, Ultra-0.5, Millipore) composed of low-protein-binding regenerated cellulose to remove lipids and proteins. Filtration was performed by a first centrifugation for $15 \mathrm{~min}$ at $16000 \mathrm{~g}$ followed by a second centrifugation in the cellulose filter centrifuge tubes for $15 \mathrm{~min}$ at $14000 \mathrm{~g}$.

\section{Sample Preparation for ${ }^{1} \mathrm{H}$ NMR Metabolic Profiling}

The final optimized sample preparation protocol was applied to $100 \mathrm{mg}$ of fecal sample aliquots of seven infants (same five infants as described above and two additional infants) collected at three time points of 8 weeks, 4 months, and 9 months of age. The optimal protocol consisted of extracting the fecal sample with UPLC-grade water with a dilution ratio of $1: 5$ feces-towater, bead beating for $10 \mathrm{~s}$ at $4 \mathrm{~m} / \mathrm{s}$ with two zirconia beads of $0.5 \mathrm{~mm}$, and a $30 \mathrm{~min}$ centrifugation at $16000 \mathrm{~g}$ at $4{ }^{\circ} \mathrm{C}$. A volume of a $400 \mu \mathrm{L}$ supernatant was stored at $-20{ }^{\circ} \mathrm{C}$ until analysis the next day.

\section{${ }^{1} \mathrm{H}$ NMR Analysis}

Fecal supernatant was thawed at room temperature (RT) followed by centrifugation for $5 \mathrm{~min}$ at $16000 \mathrm{~g}$ at $4{ }^{\circ} \mathrm{C}$. A volume of a $300 \mu \mathrm{L}$ supernatant was added to $200 \mu \mathrm{L}$ of $20 \times$ phosphate buffer $\left(200 \mathrm{mM} \mathrm{Na}_{2} \mathrm{HPO}_{4}\right.$ and $\mathrm{NaH}_{2} \mathrm{PO}_{4}, \mathrm{pH} 7.4$ at $\left.25^{\circ} \mathrm{C}\right)$ and $100 \mu \mathrm{L}$ deuterium oxide $\left(\mathrm{D}_{2} \mathrm{O}, 99.9\right.$ atom $\% \mathrm{D}$, 100G, Sigma-Aldrich, Germany), containing $1 \mathrm{mM} 3$ trimethylsilyl-tetradeutero-2,2,3,3-propionic acid (TSP- $\mathrm{d}_{4}, 98$ atom $\%$ D, Sigma-Aldrich, Germany) and $2 \mathrm{mM}$ sodium azide $\left(\mathrm{NaN}_{3}\right)$. Fecal water samples were mixed and transferred into $5 \mathrm{~mm}$ NMR tubes (Bruker Wilmad, Sigma-Aldrich, Germany), and ${ }^{1} \mathrm{H}$ NMR spectra were recorded at $298 \mathrm{~K}$ on a Bruker Avance III HD $700 \mathrm{MHz}$ (16.4 Tesla) NMR spectrometer (Bruker Biospin, Germany) equipped with a TCI cryoprobe. To account for experimental variability, samples were measured in random order. To exclude interoperator variability, samples were prepared, measured, and postprocessed by the same operator. Spectra were acquired using a one-dimensional NOESY-presat pulse sequence $\left(\mathrm{RD}-90^{\circ}\right.$-t$90^{\circ}$-tm- $90^{\circ}$-ACQ $)$ with an acquisition time of $2 \mathrm{~s}$, a relaxation delay (D1) of $5 \mathrm{~s}$, a mixing time (D8) of $100 \mathrm{~ms}$, a receiver gain of 32, 64 scans, $45 \mathrm{~K}$ data points, and a spectral width of $11161 \mathrm{~Hz}$ (15.934 ppm). Spectra were baseline-corrected, phased, calibrated, and normalized to internal TSP $(\delta 0.000$ 
ppm) using Bruker TopSpin 3.2 software. Further preprocessing and conversion of spectra into a statistical matrix were performed using an in-house script of MATLAB (https:// github.com/MUMC-MEDMIC/NMR_Preprocess MATLAB) (version R2018a, Mathworks, Natick, MA). Spectra were baseline-corrected using the asymmetric leastsquares technique; regions without peaks and regions containing water (4.71-4.95 ppm) were removed. Spectra were aligned and normalized to probabilistic quotient normalization. ${ }^{18}$ Peaks were integrated using the adaptive, intelligent binning algorithm that attempts to split the spectra so that each area common to all spectra contains the same resonance, i.e., belonging to the same metabolite. The width of each area is then determined by the maximum difference of chemical shift among all spectra. ${ }^{19}$ Metabolites were identified using information found in the literature ${ }^{14,17,20}$ or derived from the Human Fecal Metabolome Database (https:// fecalmetabolome.ca/) and Chenomx NMR Suite (version 8.5, Alberta, Canada).

\section{Data Analysis}

To investigate the influence of different sample preparation protocols on the infant fecal metabolome, a visual comparison of the NMR spectra was performed. The signal-to-noise ratio (SNR) was calculated as the ratio of the peak height (the reference peak of alanine at $1.477 \mathrm{ppm}$ ) over the root-meansquare deviation value of the noise floor. The coefficient of variation (\% CV) was calculated as an index of the reproducibility performance of metabolites using data obtained from two aliquots prepared from feces of the same individual. Multivariate statistical modeling was performed using SIMCA$\mathrm{P}^{+} 15$ (Umetrics, Umeå, Sweden). The obtained integration values were log-transformed and Pareto-scaled. Unsupervised principal component analysis (PCA) was performed to assess the variation between fecal samples based on the NMR spectra. Supervised orthogonal projections to latent structure discriminant analysis (OPLS-DA) were applied to compare ${ }^{1} \mathrm{H}$ NMR metabolic profiles of consecutive samples of five infants collected at 8 weeks, 4 , and 9 months of age. The loading plot indicates which variables (or annotated metabolites) are most accountable for these differences. Variables with an importance for the projection (VIP) value exceeding 1.5 were considered to be most important in both explaining $\mathrm{X}$ and correlating to Y. To avoid model overfitting and assure validity of the constructed OPLS-DA classification model, the analyses were repeated after adding the ${ }^{1} \mathrm{H}$ NMR metabolic profile of two additional subjects as an independent validation set.

\section{RESULTS AND DISCUSSION}

Inconsistencies in fecal sample collection, preparation, storage, and analyses can all contribute to changes in enzyme activity, high reactivity of metabolites, or breakdown or degradation of metabolites resulting in undesired changes in the metabolite content of a sample. Therefore, a robust analytical preparation protocol is required to minimize metabolite variations caused by variations during sample handling.

Determination of the Optimal Extraction Method for Gut Microbiota-Derived Metabolites

Extraction of fecal samples is essential in metabolomics for obtaining reliable and reproducible results. The extraction method should be optimized in such a way that it extracts the maximum possible number of metabolites and the procedure does not alter the identity or the amount of the metabolites to be extracted. The influence of using either UPLC-grade water or phosphate buffer for the extraction of fecal metabolites was evaluated by assessing the metabolite compositions in the NMR profile obtained from the different extracts. A comparison of such typical ${ }^{1} \mathrm{H}$ NMR spectra (region 0.5-1.6 $\mathrm{ppm}$ ) of feces, extracted with UPLC-grade water or phosphate buffer, is shown in Figure 2A. With respect to metabolite concentrations, fecal samples extracted with water show higher peaks and therefore indicate a better recovery and extraction efficiency of short-chain fatty acids (SCFA: $n$-butyrate, isobutyrate, and propionate) than found in samples extracted with phosphate buffer. Other researchers ${ }^{21}$ compared the efficiency of metabolite extraction by water and methanol and showed a better recovery of SCFAs when extracting with water. On the other hand, methanol is preferred for the extraction of lipids, malate, and cholate. Methanol, but also acetonitrile or mixed-solvent solutions such as methanol/chloroform/water, is mostly used for the identification of lipids and other hydrophobic molecules, and these organic solvent-based extractions are time consuming, labor-intensive, expensive, and hazardous to use. On the basis of our results, and supported by findings in the literature, we can conclude that the extraction efficiency is metabolite-dependent and that the recommended extraction solvent for obtaining SCFAs in infant feces is UPLC-grade water.

Not only the choice of solvent for extraction of feces is important but also the volume used, i.e., the dilution ratio, can impact the final results. A major challenge for this study is that feces collected from newborns and young infants are often limited in volume compared with adults' feces. The use of diapers adds to this because they have the incredible ability to absorb liquid feces that becomes lost and cannot be extracted back. Therefore, parents are asked to scoop up as much feces as possible from the diaper and make use of a liner that can prevent absorption as much as possible. These conditions have to be the same for all studied subjects because differences in the way of collection can introduce additional analytical bias. The minimal amount of feces to obtain reproducible results for NMR-based metabolomics in a feasible way is $100 \mathrm{mg}$. ${ }^{17,22}$

In the present study, the SNR and reproducibility of two dilution ratios, i.e., $1: 5$ and 1:10 feces-to-water, were investigated. The ${ }^{1} \mathrm{H}$ NMR spectra (region $0.8-3 \mathrm{ppm}$ ) obtained from an individual sample with each spectrum corresponding to different dilution ratios, are shown in Figure S1. The dilution ratio 1:5 shows double the signal intensities of the dilution ratio 1:10; however, the calculated SNR (mean \pm SD: $95.1 \pm 43.1$ vs $99.9 \pm 45.4$, respectively) did not appear to be significantly different between the two dilutions (Figure S1). Principally, concentrated samples are beneficial in NMR spectroscopy, as the SNR will increase with increasing amount of compound (see Figure S1). However, one has to be aware that concentrated samples at the same time may lack signal stability due to $\mathrm{pH}$ variations and intermolecular interactions. ${ }^{14}$ Therefore, the reproducibility performance of the metabolites extracted in different dilutions was also examined by analyzing the coefficient of variation (\%CV) of seven different metabolites extracted from fecal samples of one individual (Figure 2B). A threshold of $\leq 15 \% \mathrm{CV}$ is defined as most optimal. ${ }^{23}$ Metabolites exceeding this threshold are considered unstable for a robust and reproducible analysis. Choline $(>15 \%)$, succinate $(\approx 20 \%)$, iso-valerate $(>15 \%)$, and $n$-butyrate $(\approx 20 \%)$ extracted at a dilution ratio of $1: 10$ show a $\% \mathrm{CV}$ higher than 15 when compared to metabolites extracted 
with the 1:5 dilution ratio. Hence, we suggest that a dilution ratio of 1:5 feces-to-solvent is recommended as a more diluted solution will affect the reproducibility, and therefore detection and quantification, of most gut microbiota-derived metabolites. On multivariate level, however, it shows that interindividual differences still outperform the effect of extraction solvent and dilution (Figure S2).

In addition to choosing the right dilution factor to achieve the greatest reproducibility, the right choice of statistical normalization is also important to reduce the effect of intrinsic dilution (water content of feces) and clear interindividual differences between infants. Probabilistic quotient normalization (PQN) with reference to the median spectrum can largely resolve the effect of dilution on spectroscopic profiles. ${ }^{24}$ Some researchers use lyophilization as a method to minimize the effect of water content of feces. Unfortunately, lyophilization of feces, not adjusted to $\mathrm{pH} \mathrm{7,} \mathrm{affects} \mathrm{the} \mathrm{concentration} \mathrm{of}$ volatile metabolites, such as SCFAs. ${ }^{21,25}$ Therefore, and because lyophilization is an additional time-consuming step, fresh fecal samples are considered the preferred choice for fecal metabolomics studies.

\section{Assessment of Different Homogenization Methods and} Particulate Removal

Homogenization of feces is necessary to ensure that all metabolites are evenly distributed in each fecal fraction so that analytical biases due to the heterogeneity of fecal samples can be avoided. In addition, applying homogenization in fecal sample preparation aims at a more efficient extraction by enabling a better penetration of extraction solvent throughout the sample. The most commonly used homogenization methods for sample preparation of feces are vortex shaking, sonication, and bead beating. ${ }^{17,22,26}$ Sonication, however, affects the fecal metabolic profile as it lowers the relative contribution of SCFAs to the spectral profile according to a ${ }^{1} \mathrm{H}$ NMR study performed in rats. ${ }^{25}$ Hence, physical disruption using thorough mixing or bead-based grinding is preferred. In this study, the impact of homogenization by vortex shaking or bead beating on the infant fecal metabolome was evaluated. Superimposed ${ }^{1} \mathrm{H}$ NMR spectra (region 0.8-1.6 ppm), corresponding to vortex shaking and bead beating, is shown in Figure 2C. With respect to metabolite concentrations, the spectra are near-identical despite small chemical shift changes for some specific metabolites. Samples homogenized using bead beating show a better recovery of $n$-butyrate, branchedchain amino acids (BCAAs: valine, leucine, and isoleucine), alanine, lactate, threonine, and ethanol than samples that were vortexed. The SNR measured from the fecal extracts with different homogenization methods shows that extracts subjected to bead beating have a considerably higher SNR in comparison to vortexed (reference) extracts (Figure S1B). This difference was, however, proven to be statistically insignificant, probably due to the lower number of samples studied. Other important aspects to consider are the feasibility, time, and yield of the protocol. When using bead beating instead of vortex shaking, it shows that samples that are processed over a very short period lead to a higher yield of extracted metabolites. In addition, samples processed by bead beating show a clearer supernatant, which is an important prerequisite to ensure high-quality ${ }^{1} \mathrm{H}$ NMR spectra. ${ }^{11}$

The last step before the actual preparation for ${ }^{1} \mathrm{H}$ NMR analyses is the removal of particulates to obtain highly reproducible, high-resolution spectra leading to more accurate signal intensities. On the one hand, the influence of centrifugation duration on the metabolome was investigated and, on the other hand, the impact of using different filtration concentration factors was evaluated. Typical ${ }^{1} \mathrm{H}$ NMR spectra $(0.5-1.6 \mathrm{ppm})$ of fecal extracts subjected to a different duration of centrifugation are depicted in Figure 2D. Fecal extracts centrifuged for $30 \mathrm{~min}$ show a higher yield of metabolites as compared to samples centrifuged for only 15 min. This finding can be explained by the fact that more cellular components are removed after $30 \mathrm{~min}$ as compared to 15 min centrifugation time. This results in a clearer supernatant and consequently higher-quality ${ }^{1} \mathrm{H}$ NMR spectra. The removal of cellular components can also result in a reduction of various biological and chemical processes within the sample, hence minimizing their impact on the biochemical composition of the sample. ${ }^{29}$ When centrifuge filter tubes are used to remove particulate, unwanted peaks of glycerol arise in the ${ }^{1} \mathrm{H}$ NMR spectrum (Figure S3). To minimize the influence of glycerol that is released from the filter tubes itself, the tubes can be prewashed with sterile water. However, this is a very time-consuming treatment, something that does not contribute to the development of a practical protocol. Next to the glycerol peaks, amino acids, BCAAs, and SCFAs extracted from samples filtrated with $30 \mathrm{kDa}$ tubes are better recovered as compared to those filtrated with 10 and $3 \mathrm{kDa}$ filter tubes. In general, filtration of fecal extract shows lower signal intensities of these metabolites in the ${ }^{1} \mathrm{H}$ NMR spectrum as compared to centrifugation only. From a multivariate point of view, it shows that intraindividual variation caused by differences in the filtration concentration factor surpasses interindividual variation (Figure S4).

\section{Stability of the Fecal Metabolome}

During ${ }^{1} \mathrm{H}$ NMR acquisition, metabolites might change over time due to either enzymatic or nonenzymatic processes that take place within the sample. It is therefore essential to elucidate spectral changes that might occur over time. ${ }^{1} \mathrm{H}$ NMR spectra of a pooled sample were acquired over a period of $5 \mathrm{~h}, 19 \mathrm{~h}, 30 \mathrm{~h}$, and 1 week and visually analyzed by overlaying the obtained spectra (Figure S5). Significant changes are observed when samples are analyzed after 1 week at room temperature. Especially the concentration and chemical shift values of acidic metabolites are influenced. The spectral region between 1.25 and $1.35 \mathrm{ppm}$ also shows great differences between samples analyzed after $5 \mathrm{~h}$ and those analyzed after 1 week, 30, and 19 h. No significant effect was observed between the reference $(0 \mathrm{~h}$ on RT) and samples analyzed after $5 \mathrm{~h}$ at room temperature. The PCA score plot confirms that the samples analyzed after $5 \mathrm{~h}$ resemble the reference samples and greatly deviate from samples analyzed after $19 \mathrm{~h}, 30 \mathrm{~h}$, and 1 week. The longer the sample stays at room temperature, the more acidification takes place by bacterial enzymes (e.g., increase in lactic acid and buildup of related acidic metabolites). To summarize, ${ }^{1} \mathrm{H}$ NMR experiments are ideally performed within a time frame of a few minutes to a couple of hours.

Proposed Protocol for Sample Preparation for ${ }^{1} \mathrm{H}$ NMR Metabolic Phenotyping of Infant Feces

${ }^{1} \mathrm{H}$ NMR spectroscopy is chosen over gas chromatography (GC) - and liquid chromatography-mass spectrometry (LCMS) as analytical tool for fecal metabolic phenotyping. ${ }^{28}$ Metabolomics on infant feces, however, is still understudied, and previously described methods for ${ }^{1} \mathrm{H}$ NMR sample 


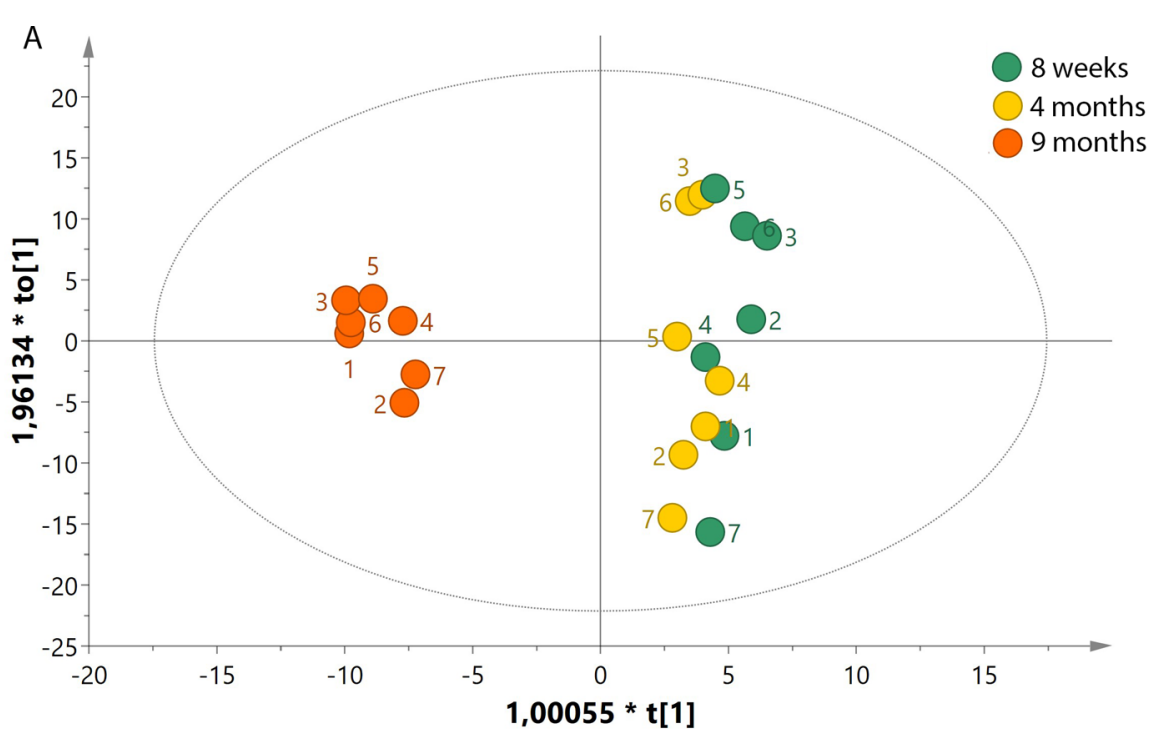

\section{B}

(d) 9 months

breast milk, formula milk and solid foods

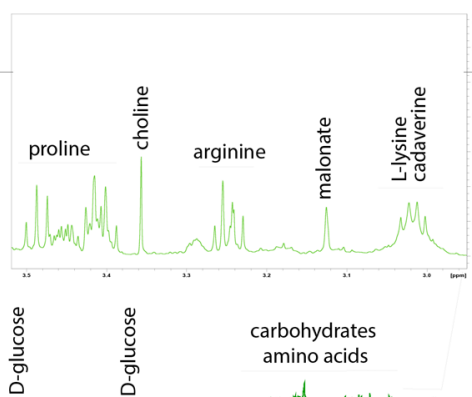

?
$=$
$=$
$=$
$=$
$=$

(c) 4 months
breast milk and solid foods

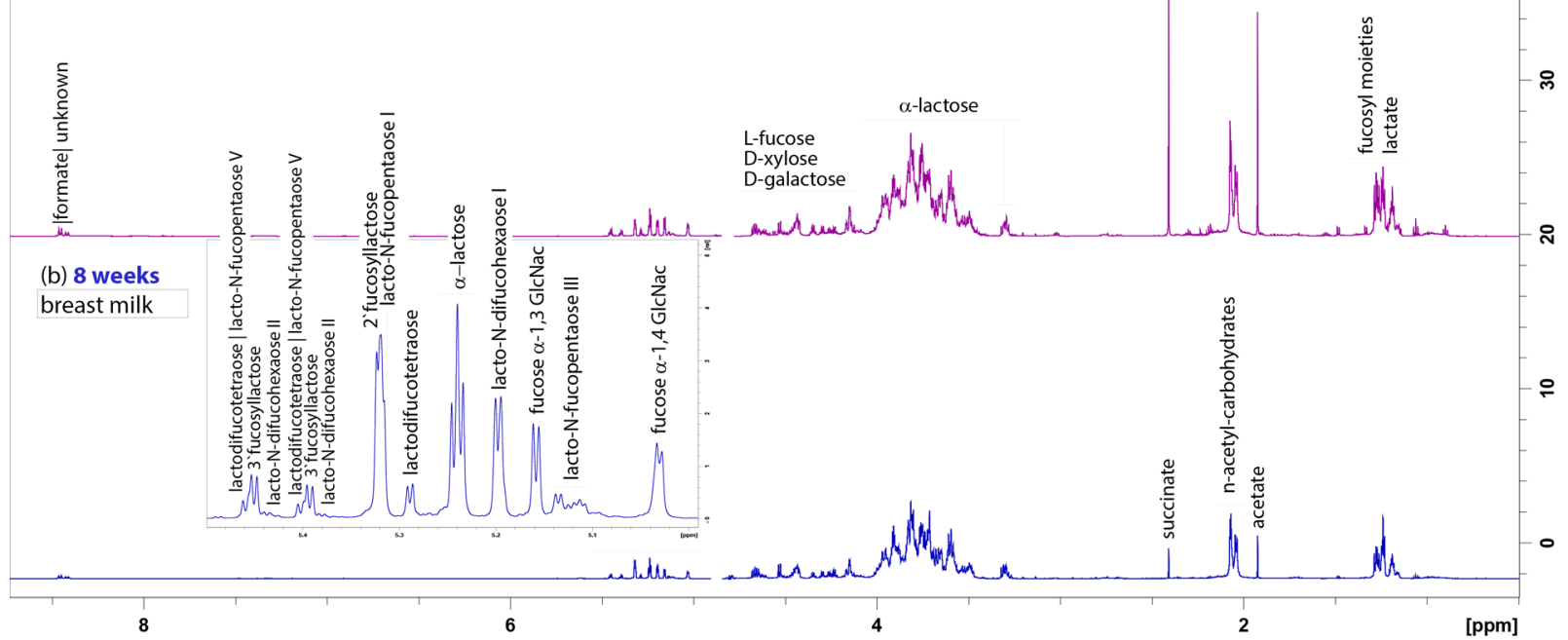

(a) 8 weeks
formula milk

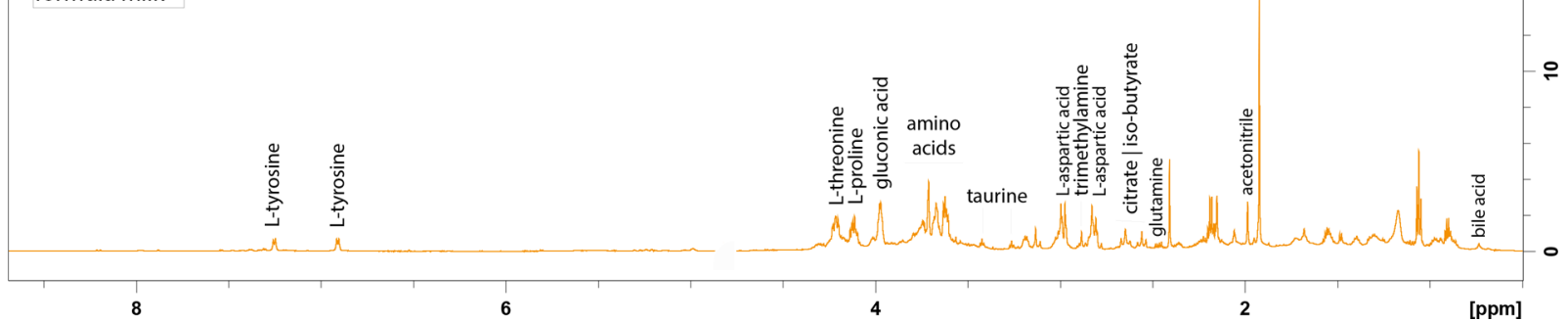

Figure 3. OPLS-DA score plot of infant fecal metabolic profiles when classified by age 8 weeks, 4 months, and 9 months (A). Each number (1-7) represents an observation of the respective subject. Infant fecal ${ }^{1} \mathrm{H}$ NMR spectra showing the age variability from 8 weeks to 9 months postpartum 
Figure 3. continued

(B) for an 8 week formula-fed infant (a) and an 8 week breast-fed (b), 4 month breast- and solid-fed (c), and 9 month breast-, formula-, and solidfed infant (d). ${ }^{1} \mathrm{H}$ NMR spectra (region $0.5-8.5 \mathrm{ppm}$ ) derived from fecal samples of subjects 7 (a) and 6 (b-d) processed according to the proposed protocol.

preparation vary greatly. $22,29-33$ Besides this variation in preparation methods, pretreatment of samples such as sonication, lyophilization, and freeze-drying is studied. It was previously shown that these handlings lead to a lower relative contribution of SCFAs to the final spectral profile. ${ }^{20,25}$ Filtration of fecal supernatants results in a clean, particulatefree extract and can obtain improved shimming of NMR spectrometer and ensure a better quality of the NMR spectrum. ${ }^{28}$ Unfortunately, this latter study showed that the filtration of the sample can add significant experimentalinduced artifacts, i.e., unwanted glycerol peaks derived from the filter. Therefore, we strongly discouraged to use filter tubes for particulate removal as it can greatly influence the fecal metabolic profile of infants leading to incorrect interpretations. Another drawback of using filters is that they require larger sample volumes as some volume is lost during filtration. This is not optimal when working with small volumes of infant feces.

The proposed protocol of this study took into account the feasibility, fecal metabolite yield, and recovery, making it suitable as an agile and robust reference (Supporting Information). In the near future, however, the effect of water content and diurnal variation of feces has to be explored too, as it might have an impact on the final interpretation of the results. This protocol will ultimately contribute to a reliable comparison, confirmation, and validation of study findings reported by other researchers in the field.

\section{${ }^{1} \mathrm{H}$ NMR Metabolic Phenotyping of Infant Feces}

The developed protocol was applied on fecal samples of infants to study the development of the gut metabolome. Multivariate analyses show that the individual fecal metabolome of infants aged 8 weeks resembles that of 4 months but is clearly different from the metabolome at 9 months of age (Figure 3A). This difference is mainly driven by an increasing concentration of $n$ butyrate and BCAAs toward 9 months of age. When the infants' diet switches to solid foods, $n$-butyrate is prevailing as the product of bacterial fermentation of indigestible carbohydrates and BCAAs are derived from dietary proteins. At 8 weeks and 4 months, the levels of milk oligosaccharide derivatives and lactic acid are highest. Feces of breast-fed infants are rich in human milk oligosaccharides (HMOs), whereas formula-fed infants show a higher amount of amino acids derived from proteins present in formula milk (Figure 3B). At 9 months, after solid food is introduced, interindividual variation becomes less pronounced and the metabolite composition develops into a mature metabolome (Figure $3 \mathrm{~A}$ ). It is clear that the observed fecal metabolites are mainly derived from the diet and bacterial fermentation in the gut. Therefore, it is highly recommended to include nutritional intake and gut microbiome analyses in future studies on the infant gut metabolome. This can provide valuable insight into the interactions between nutrition, gut microbiota, and metabolites shaping infants' health in the first year of life.

\section{CONCLUSIONS}

This work provides evidence on preanalytical-induced alterations in the biochemical composition of feces and proposes a hands-on and robust sample preparation protocol for ${ }^{1} \mathrm{H}$ NMR metabolic phenotyping of infant gut health. Ongoing research will enable us to develop one reference model of fecal metabolomics in healthy infants. The present study may define an essential starting point, providing valuable insights into the association between metabolic profile and physiological infant maturation. Fecal metabolites obtained from infants at weeks and months of age can be considered as crucial biomarkers to monitor infants' gut health development in longitudinal large-scale multiomics studies.

\section{ASSOCIATED CONTENT}

\section{Supporting Information}

The Supporting Information is available free of charge at https://pubs.acs.org/doi/10.1021/acs.jproteome.1c00617.

Extended methods on sample preparation and ${ }^{1} \mathrm{H}$ NMR spectral analysis; ${ }^{1} \mathrm{H}$ NMR spectra and signal-to-noise ratios from infant feces (Figure S1); PCA score plots annotated according to the subject and sample preparation method (Figure S2); ${ }^{1} \mathrm{H}$ NMR spectrum of infant fecal samples prepared with different particulate removal methods (Figure S3); PCA score annotated according to the subject and filtration tube sizes (Figure S4); PCA score plot and ${ }^{1} \mathrm{H}$ NMR spectrum of infant fecal pools measured at different times (Figure S5); and subject characteristics (Table S1) (PDF)

\section{AUTHOR INFORMATION}

Corresponding Author

John Penders - Department of Medical Microbiology, NUTRIM School of Nutrition and Translational Research in Metabolism, Maastricht University, 6229 HX Maastricht, The Netherlands; ㅇo orcid.org/0000-0001-9146-5919; Email: j.penders@maastrichtuniversity.nl

\section{Authors}

Liene Bervoets - Department of Medical Microbiology, NUTRIM School of Nutrition and Translational Research in Metabolism, Maastricht University, 6229 HX Maastricht, The Netherlands

Johannes H. Ippel - Department of Biochemistry, CARIM Cardiovascular Research Institute Maastricht, Maastricht University, 6229 HX Maastricht, The Netherlands

Agnieszka Smolinska - Department of Pharmacology and Toxicology, NUTRIM School of Nutrition and Translational Research in Metabolism, Maastricht University, 6229 HX Maastricht, The Netherlands

Niels van Best - Department of Medical Microbiology, NUTRIM School of Nutrition and Translational Research in Metabolism, Maastricht University, 6229 HX Maastricht, The Netherlands; Institute of Medical Microbiology, RWTH University Hospital Aachen, 52074 Aachen, Germany

Paul H. M. Savelkoul - Department of Medical Microbiology, NUTRIM School of Nutrition and Translational Research in Metabolism, Maastricht University, 6229 HX Maastricht, The Netherlands; Department of Medical Microbiology \& 
Infection Control, VUMC, 1081 HV Amsterdam, The Netherlands

Monique A. H. Mommers - Department of Epidemiology, Maastricht University, 6229 HX Maastricht, The Netherlands

Complete contact information is available at: https://pubs.acs.org/10.1021/acs.jproteome.1c00617

\section{Author Contributions}

The manuscript was written through contributions of all authors. All authors have given approval to the final version of the manuscript.

Notes

The authors declare no competing financial interest.

\section{ACKNOWLEDGMENTS}

The authors thank Eda Aydeniz for her practical assistance in analyzing the samples and Ronny Mohren for his assistance in providing materials for the analyses. The authors also thank all participants for their willingness to participate in this study. This study was financially supported by a grant from the Joint Programming Initiative A healthy diet for a healthy life (HDHL) Joint Action Intestinal Microbiomics (project number 50-52905-98-599). TOC graphic was created with BioRender.com.

\section{REFERENCES}

(1) Galazzo, G.; van Best, N.; Bervoets, L.; Dapaah, I. O.; Savelkoul, P. H.; Hornef, M. W.; consortium, G.-M.; Lau, S.; Hamelmann, E.; Penders, J.; et al. Development of the Microbiota and Associations With Birth Mode, Diet, and Atopic Disorders in a Longitudinal Analysis of Stool Samples, Collected From Infancy Through Early Childhood. Gastroenterology 2020, 158, 1584-1596.

(2) Stokholm, J.; Blaser, M. J.; Thorsen, J.; Rasmussen, M. A.; Waage, J.; Vinding, R. K.; Schoos, A. M.; Kunoe, A.; Fink, N. R.; Chawes, B. L.; Bonnelykke, K.; Brejnrod, A. D.; Mortensen, M. S.; AlSoud, W. A.; Sorensen, S. J.; Bisgaard, H. Maturation of the gut microbiome and risk of asthma in childhood. Nat. Commun. 2018, 9, No. 141.

(3) Galazzo, G.; Tedjo, D. I.; Wintjens, D. S. J.; Savelkoul, P. H. M.; Masclee, A. A. M.; Bodelier, A. G. L.; Pierik, M. J.; Jonkers, D.; Penders, J. Faecal Microbiota Dynamics and their Relation to Disease Course in Crohn's Disease. J. Crohns Colitis 2019, 13, 1273-1282.

(4) Ray, K. IBD. Understanding gut microbiota in new-onset Crohn's disease. Nat. Rev. Gastroenterol. Hepatol. 2014, 11, 268.

(5) Al Nabhani, Z.; Eberl, G. Imprinting of the immune system by the microbiota early in life. Mucosal Immunol 2020, 13, 183-189.

(6) Renz, H.; Adkins, B. D.; Bartfeld, S.; Blumberg, R. S.; Farber, D. L.; Garssen, J.; Ghazal, P.; Hackam, D. J.; Marsland, B. J.; McCoy, K. D.; Penders, J.; Prinz, I.; Verhasselt, V.; von Mutius, E.; Weiser, J. N.; Wesemann, D. R.; Hornef, M. W. The neonatal window of opportunity-early priming for life. J. Allergy Clin. Immunol. 2018, 141, 1212-1214.

(7) Costello, E. K.; Stagaman, K.; Dethlefsen, L.; Bohannan, B. J.; Relman, D. A. The application of ecological theory toward an understanding of the human microbiome. Science 2012, 336, 12551262.

(8) Martínez, I.; Muller, C. E.; Walter, J. Long-term temporal analysis of the human fecal microbiota revealed a stable core of dominant bacterial species. PLoS One 2013, 8, No. e69621.

(9) Wörheide, M. A.; Krumsiek, J.; Kastenmuller, G.; Arnold, M. Multi-omics integration in biomedical research - A metabolomicscentric review. Anal. Chim. Acta 2021, 1141, 144-162.
(10) Li, D.; Gao, C.; Zhang, F.; Yang, R.; Lan, C.; Ma, Y.; Wang, J. Seven facts and five initiatives for gut microbiome research. Protein Cell 2020, 11, 391-400.

(11) Keun, H. C. NMR-Based Metabolomics; Royal Society of Chemistry: Cambridge, UK, 2018; pp 341-343.

(12) Zierer, J.; Jackson, M. A.; Kastenmuller, G.; Mangino, M.; Long, T.; Telenti, A.; Mohney, R. P.; Small, K. S.; Bell, J. T.; Steves, C. J.; Valdes, A. M.; Spector, T. D.; Menni, C. The fecal metabolome as a functional readout of the gut microbiome. Nat. Genet. 2018, 50, $790-795$.

(13) Karu, N.; Deng, L.; Slae, M.; Guo, A. C.; Sajed, T.; Huynh, H.; Wine, E.; Wishart, D. S. A review on human fecal metabolomics: Methods, applications and the human fecal metabolome database. Anal. Chim. Acta 2018, 1030, 1-24.

(14) Lamichhane, S.; Yde, C. C.; Schmedes, M. S.; Jensen, H. M.; Meier, S.; Bertram, H. C. Strategy for Nuclear-Magnetic-ResonanceBased Metabolomics of Human Feces. Anal. Chem. 2015, 87, 59305937.

(15) van Best, N.; Hornef, M. W.; Savelkoul, P. H.; Penders, J. On the origin of species: Factors shaping the establishment of infant's gut microbiota. Birth Defects Res., Part C 2015, 105, 240-251.

(16) de Korte-de Boer, D.; Mommers, M.; Creemers, H. M.; Dompeling, E.; Feron, F. J.; Gielkens-Sijstermans, C. M.; Jaminon, M.; Mujakovic, S.; van Schayck, O. C.; Thijs, C.; Jansen, M. LucKi Birth Cohort Study: rationale and design. BMC Public Health 2015, 15, No. 934.

(17) Amiot, A.; Dona, A. C.; Wijeyesekera, A.; Tournigand, C.; Baumgaertner, I.; Lebaleur, Y.; Sobhani, I.; Holmes, E. (1)H NMR Spectroscopy of Fecal Extracts Enables Detection of Advanced Colorectal Neoplasia. J. Proteome Res. 2015, 14, 3871-3881.

(18) Dieterle, F.; Ross, A.; Schlotterbeck, G.; Senn, H. Probabilistic quotient normalization as robust method to account for dilution of complex biological mixtures. Application in $1 \mathrm{H}$ NMR metabonomics. Anal. Chem. 2006, 78, 4281-4290.

(19) De Meyer, T.; Sinnaeve, D.; Van Gasse, B.; Tsiporkova, E.; Rietzschel, E. R.; De Buyzere, M. L.; Gillebert, T. C.; Bekaert, S.; Martins, J. C.; Van Criekinge, W. NMR-based characterization of metabolic alterations in hypertension using an adaptive, intelligent binning algorithm. Anal. Chem. 2008, 80, 3783-3790.

(20) Kostidis, S.; Kokova, D.; Dementeva, N.; Saltykova, I. V.; Kim, H. K.; Choi, Y. H.; Mayboroda, O. A. (1)H-NMR analysis of feces: new possibilities in the helminthes infections research. BMC Infect. Dis. 2017, 17, No. 275.

(21) Jacobs, D. M.; Deltimple, N.; van Velzen, E.; van Dorsten, F. A.; Bingham, M.; Vaughan, E. E.; van Duynhoven, J. (1)H NMR metabolite profiling of feces as a tool to assess the impact of nutrition on the human microbiome. NMR Biomed 2008, 21, 615-626.

(22) Bridgman, S. L.; Azad, M. B.; Field, C. J.; Haqq, A. M.; Becker, A. B.; Mandhane, P. J.; Subbarao, P.; Turvey, S. E.; Sears, M. R.; Scott, J. A.; Wishart, D. S.; Kozyrskyj, A. L. Investigators, C. S. Fecal ShortChain Fatty Acid Variations by Breastfeeding Status in Infants at 4 Months: Differences in Relative versus Absolute Concentrations. Front. Nutr. 2017, 4, No. 11.

(23) Louis, E.; Bervoets, L.; Reekmans, G.; De Jonge, E.; Mesotten, L.; Thomeer, M.; Adriaensens, P. Phenotyping human blood plasma by 1 H-NMR: a robust protocol based on metabolite spiking and its evaluation in breast cancer. Metabolomics 2015, 11, 225-236.

(24) Jackson, F.; Georgakopoulou, N.; Kaluarachchi, M.; Kyriakides, M.; Andreas, N.; Przysiezna, N.; Hyde, M. J.; Modi, N.; Nicholson, J. K.; Wijeyesekera, A.; Holmes, E. Development of a Pipeline for Exploratory Metabolic Profiling of Infant Urine. J. Proteome Res. 2016, 15, 3432-3440.

(25) Saric, J.; Wang, Y.; Li, J.; Coen, M.; Utzinger, J.; Marchesi, J. R.; Keiser, J.; Veselkov, K.; Lindon, J. C.; Nicholson, J. K.; Holmes, E. Species variation in the fecal metabolome gives insight into differential gastrointestinal function. J. Proteome Res. 2008, 7, 352360 . 
(26) Matysik, S.; Le Roy, C. I.; Liebisch, G.; Claus, S. P. Metabolomics of fecal samples: a practical consideration. Trends Food Sci. Technol. 2016, 57, 244-255.

(27) Gratton, J.; Phetcharaburanin, J.; Mullish, B. H.; Williams, H. R.; Thursz, M.; Nicholson, J. K.; Holmes, E.; Marchesi, J. R.; Li, J. V. Optimized Sample Handling Strategy for Metabolic Profiling of Human Feces. Anal. Chem. 2016, 88, 4661-4668.

(28) Deda, O.; Gika, H. G.; Wilson, I. D.; Theodoridis, G. A. An overview of fecal sample preparation for global metabolic profiling. $J$. Pharm. Biomed. Anal. 2015, 113, 137-150.

(29) Martin, F.-P. J.; Moco, S.; Montoliu, I.; Collino, S.; Da Silva, L.; Rezzi, S.; Prieto, R.; Kussmann, M.; Inostroza, J.; Steenhout, P. Impact of breast-feeding and high- and low-protein formula on the metabolism and growth of infants from overweight and obese mothers. Pediatr. Res. 2014, 75, 535-543.

(30) Shankar, V.; Homer, D.; Rigsbee, L.; Khamis, H. J.; Michail, S.; Raymer, M.; Reo, N. V.; Paliy, O. The networks of human gut microbe-metabolite associations are different between health and irritable bowel syndrome. ISME J 2015, 9, 1899-1903.

(31) Del Chierico, F.; Vernocchi, P.; Petrucca, A.; Paci, P.; Fuentes, S.; Pratico, G.; Capuani, G.; Masotti, A.; Reddel, S.; Russo, A.; Vallone, C.; Salvatori, G.; Buffone, E.; Signore, F.; Rigon, G.; Dotta, A.; Miccheli, A.; de Vos, W. M.; Dallapiccola, B.; Putignani, L. Phylogenetic and Metabolic Tracking of Gut Microbiota during Perinatal Development. PLoS One 2015, 10, No. e0137347.

(32) Kim, H. K.; Rutten, N. B.; Besseling-van der Vaart, I.; Niers, L. E.; Choi, Y. H.; Rijkers, G. T.; van Hemert, S. Probiotic supplementation influences faecal short chain fatty acids in infants at high risk for eczema. Benefic Microbes 2015, 6, 783-790.

(33) He, X.; Parenti, M.; Grip, T.; Lonnerdal, B.; Timby, N.; Domellof, M.; Hernell, O.; Slupsky, C. M. Fecal microbiome and metabolome of infants fed bovine MFGM supplemented formula or standard formula with breast-fed infants as reference: a randomized controlled trial. Sci. Rep. 2019, 9, No. 11589. 